\title{
NECROLOGI
}

\section{RICORDO DI SERGIO FRUGIS}

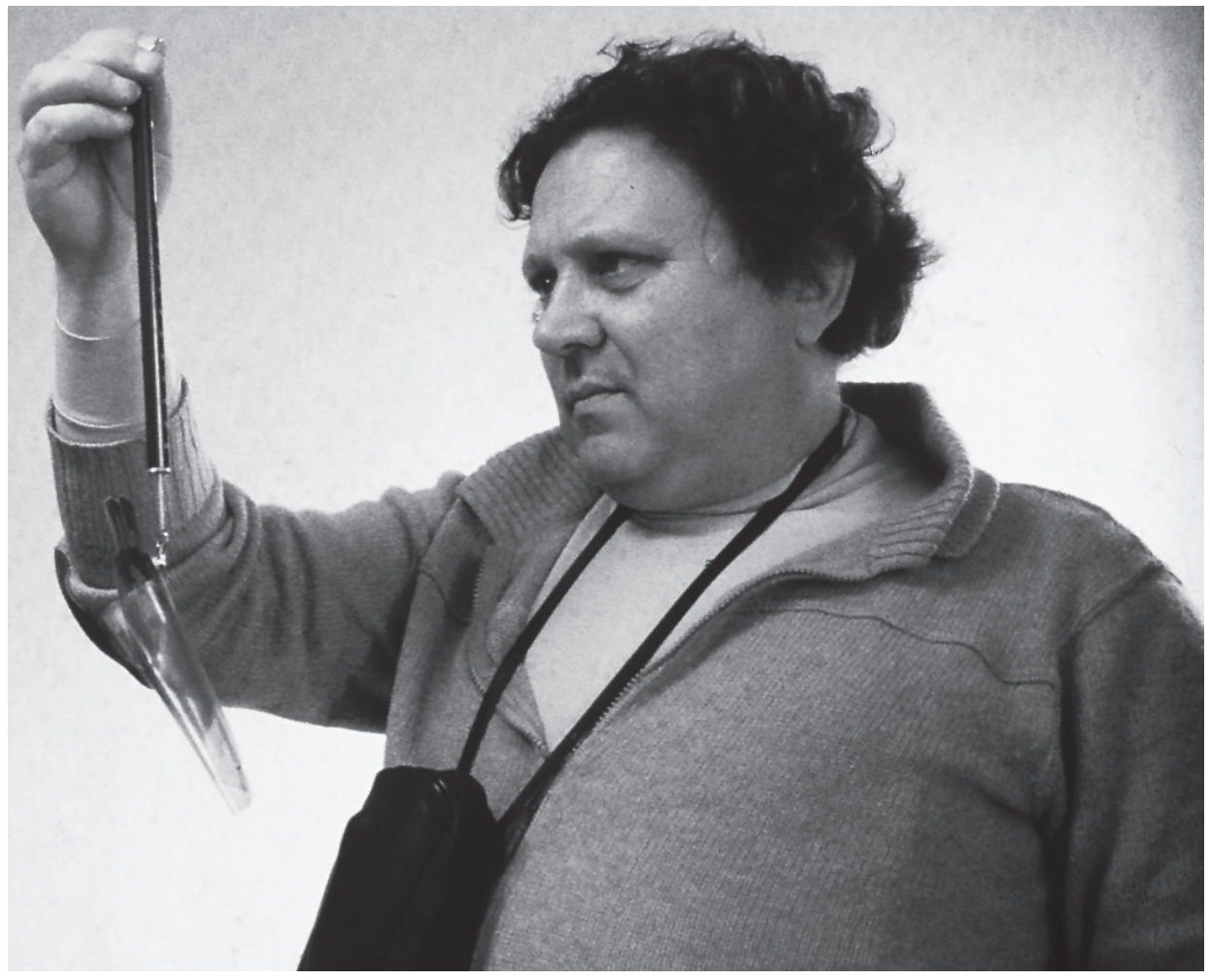

Sergio Frugis (6 ottobre 1930 - 13 settembre 2009) 
Quando ho avuto la notizia della scomparsa di Sergio Frugis, dopo tanti anni di sofferenza fisica e psichica, ho pensato subito che l'ornitologia italiana gli doveva qualcosa; fu lui a promuovere le prime timide riunioni tra ornitologi all'UZI (ricordo Ferrara nel 1977 di cui c'è un resoconto a pag. 48 del $\mathrm{n}^{\circ} 1$ di Avocetta del 1978; e ancora Catania nel 1979), la fondazione del Centro Italiano Studi Ornitologici (CISO) (1976) e la rivista Avocetta.

Questa rivista era nata come un ciclostilato che girava tra amici nel 1953, ma divenne una testata a tutti gli effetti dopo la costituzione del CISO. I congressi di ornitologia sono stati una sua idea, che poi è divenuta una tradizione biennale (il primo organizzato ad Aulla nel 1981 e il secondo organizzato a Parma nel 1983 proprio da Frugis e i suoi allievi).

Almeno gli ultimi trenta anni della vita di Sergio Frugis sono stati davvero pesanti; prima la lunga e lenta malattia della moglie Lucia, fino alla dolorosa scomparsa, poi il suo implacabile isolamento dovuto ad un peggioramento delle sue condizioni fisiche che lo ha costretto a un ricovero in ospedale fino alla fine. Il destino è stato davvero ostile nei confronti di Sergio, che amava conversare, raccontare e tenere rapporti e interazioni con il mondo esterno. Aveva perso la possibilità di parlare e di esprimersi, ma poteva solo ascoltare, capire quello che gli si diceva, senza avere la possibilità di replica.

Chi gli è stato vicino racconta dell'intensità del suo sguardo, dell'attenzione che riservava ai visitatori che si sforzavano in ogni modo di alleggerire la tragedia del monologo e della disperazione che traspariva da quello sguardo profondo e ancora vigile. Ha avuto la mente lucida fino agli ultimi giorni, il suo cervello era in perfette condizioni, solo il corpo aveva ceduto; quando le persone più a lui vicine gli portavano i saluti di amici e colleghi restituiva il saluto con un piccolo cenno della mano, come per dire "ciao". Si è spento improvvisamente la mattina di una domenica, pochi giorni prima di compiere il suo $79^{\circ}$ anno.

Nel 1986 partecipai al congresso internazionale sugli Atlanti a Dijon; avevo sentito per telefono Sergio Frugis che mi aveva proposto di profittare di un passaggio con lui in auto da Parma, cosa che feci con molto piacere. Al gruppo si aggiunse a Torino anche Toni Mingozzi. Ricordo che il viaggio fu molto lungo, di tanto in tanto facevamo qualche sosta, ma Sergio era un pilota automatico davvero instancabile; fumava sigarette di continuo ed era anche una buona forchetta. Durante il viaggio ci raccontò quasi tutta la sua vita, tanti episodi di ornitologia, molte ricerche svolte da amici stranieri su tantissime specie, insomma era un'enciclopedia viaggiante.

Ho un bellissimo e nitido ricordo di quel viaggio a Dijon, andata e ritorno con chi aveva fatto traghettare l'ornitologia da una scienza che studia solo gli esemplari dei musei ad una che si cimenta anche 
nell'analisi delle comunità. Nello stesso anno, fui in contatto con il British Ornithologists' Union per realizzare la checklist degli uccelli di Sicilia; l'editor della serie delle Checklist, James Monk, scrisse a Frugis per avere informazioni sulla mia affidabilità. Questo è stato per me un segno tangibile della stima di cui godeva Sergio fuori dall'Italia.

Si batté in ogni modo per l'abolizione dell'uccellagione, retaggio antico che stentava ad essere soppresso ancora negli anni '80; dimostrò con dati di fatto (quelli pubblicati dai roccoli in Lombardia) che l'inanellamento doveva essere svolto con altri metodi se voleva essere considerato "scientifico"; ricordo che mi raccontava della sproporzione numerica di Merli femmine inanellati rispetto ai maschi, in quanto questi ultimi venivano trattenuti per il loro canto e quindi non liberati. Grazie a lui, iniziò quel processo di smantellamento o di trasformazione dei roccoli in chiave moderna, cioè scientifica.

Sergio Frugis era entrato a insegnare all'Università nel 1973 per la sua profonda passione ornitologica. Non aveva l'aspetto di un accademico e stava sempre bene con i giovani, sapendo individuare chi si distingueva nel campo ornitologico. Con Sergio non c'erano barriere, il "tu" era d'obbligo e nonostante la differenza di età tutti lo trattavano come un coetaneo e molti dei suoi allievi oggi occupano ruoli importanti in Università e Istituti di ricerca italiani.

Sergio Frugis aveva preso il meglio dalla precedente generazione di ornitologi e l'aveva saputo sfruttare in maniera moderna; nonostante Edgardo Moltoni fosse un po' scettico sulla possibilità di contare gli effettivi delle popolazioni ornitiche, Frugis lanciò in Italia i metodi di censimento già in uso in altri Paesi europei. Formulò un'idea nuova di gruppo ornitologico italiano nel CISO, all'interno del quale volle attivare tutte le iniziative ornitologiche. La prima di tutte fu la Stazione Ornitologica di Val Campotto, in cui tra il 1978 e il 1981 furono catturati e inanellati tantissimi uccelli con i metodi standardizzati in precedenza mai usati in Italia.

Sulla passerella di Val Campotto, poi passata in gestione all'allora Istituto Nazionale di Biologia della Selvaggina, si formarono moltissimi inanellatori italiani, i quali sostennero a Val Campotto anche gli esami per ottenere il permesso di inanellamento.

A Sergio Frugis dobbiamo anche l'iniziativa dell'Atlante Ornitologico Italiano realizzato purtroppo in tempi lunghissimi negli anni '80, ma con i metodi standard europei. È il caso di sottolineare che quell'opera non è mai stata ripetuta da altri.

È stato tra i primi ornitologi conservazionisti italiani e a lui e Helmar Schenk si deve la pubblicazione della prima Lista Rossa degli Uccelli d'Italia, il primo tentativo di dare un valore ai nostri uccelli. Abile disegnatore, non perdeva mai occasione durante i suoi viaggi di realizzare schizzi e disegni a matita, che poi regalava agli amici. La sua 
creazione, Avocetta, ha ospitato tantissimi suoi disegni di uccelli, che dimostrano una mano davvero esperta e sicura nella rappresentazione del suo principale diletto. E dal 2009 nella prima pagina della sua rivista campeggia la doverosa aggiunta ad Avocetta: "Journal of Ornithology founded by Sergio Frugis".

Bruno Massa

Dipartimento DEMETRA, Università degli Studi di Palermo, Viale delle Scienze - 90128 Palermo 